length as those in $\left(\mathrm{Re}_{3} \mathrm{Cl}_{12}\right)^{3-}$. This suggests a difference of bond order among the Ro-Re bonds.

On replacement of $\mathrm{Cl}$ by $\mathrm{Br}$, there is a reduction of $19^{\circ}$ in the angle subtended by the out-of-plane atoms $X(2)$ and $X(2)^{\prime}$ at the deficient $\operatorname{Re}(2)$, and the $\operatorname{Re}-\operatorname{Br}(2)$ bonds themselves are significantly shorter than any other in the bromo-anion. These observations suggest that the $\mathrm{Re}-\mathrm{Br}$ bonds to the non-deficient $\mathrm{Re}(\mathrm{l})$ are under considerable strain which is relieved on removal of one halogen atom by bond bending and also by bond shortening to what is presumably a closer approximation to a true single bond length. The first quantitative treatment of the bonding in these trinuclear anions, given by Cotton and Haas 6 , assumes that four orbitals from each rhenium atom are directed to the corners of a square and that all Re-Re bonds are equivalent. For $\left(\mathrm{Re}_{3} \mathrm{Cl}_{12}\right)^{3-}$, the only structure then known, the first assumption leads to bonds with the out-of-plane chlorine atoms which are bent by about $10^{\circ}$ only. However, relative to this scheme, the bending of these bonds in $\left(\mathrm{Re}_{3} \mathrm{Br}_{11}\right)^{2-}$ is increased markedly to $23^{\circ}$. The observations, first, of a proferonce for highly bent $\operatorname{Re}-X$ bonds and, secondly, of the non-equivalence of $R_{\theta}-R_{\theta}$ bonds, do indicate that some modification or extension of the Cotton and Haas scheme is required for $\left(\operatorname{Re}_{3} X_{11}\right)^{2-}$ ions.

The unit cell dimensions and space group of $\mathrm{Cs}_{2} \mathrm{Re}_{3} \mathrm{Br}_{11}$ were previously attributed by us (see ref. 1) to the compound $\mathrm{CsRe}_{3} \mathrm{Br}_{10}$ on the basis of bulk analyses. This was because crystals of more than one composition appear to separate under the conditions of preparation and bulk analyses are therefore misleading. We have since characterized a second crystal form from these preparations. The small, black, roughly spherical crystals are orthorhombic with cell dimensions $a=11 \cdot 1, b=14 \cdot 6, c=14.6 \AA$ and probable space group Ama2. They thus have very similar cell dimensions and the same probable space group as $\mathrm{Cs}_{3} \mathrm{Re}_{3} \mathrm{Cl}_{12}$ and are assumed to be the isostructural $\mathrm{Cs}_{3} \mathrm{Re}_{3} \mathrm{Br}_{12}$. The existence of this $\left(\mathrm{Re}_{3} \mathrm{Br}_{12}\right)^{8-}$ ion is of interest in view of the very considerable steric repulsions which must exist between bromine atoms.

We thank Dr. J. E. Fergusson and Mr. B. H. Robinson for sample preparation, and for advice. This project was supported financially by the New Zealand Universities Research Committeo.

Chemistry Department,

University of Canterbury, Christchurch, New Zealand.

1 Fergusson, J. E., and Robinson, B. H., Proc. Chem. Soc., 189 (1964).

- Fergusson, J. E., Penfold, B. R., and Robinson, W. T., Nature, 201, 181 (1964).

${ }^{3}$ Robinson, W. T., Fergusson, J. E., and Penfold, B. R., Proc. Chem. Soc., 116 (1963).

- Bertrand, J. A., Cotton, F. A., and Dollase, W. A., Inorg. Chem., 2, 1166 (1963).

- Robinson, W. T., and Penfold, B. R. (to be published).

- Cotton, F. A., and Haas, T. E., Inorg. Chem., 3, 10 (1964).

\section{Distribution of Benzcarbazole Isomers in Petroleum as Evidence for their Biogenic Origin}

A RECENT paper ${ }^{1}$ has discussed the distribution of different types of nitrogen compounds in the $200^{\circ}-500^{\circ} \mathrm{C}$ fraction from petroleums of varying geological age. Carbazoles and benzcarbazoles were found to be predominant nitrogen types in all crudes, accounting for 25-70 per cent of total nitrogen in this boiling range, and as much as 2 per cent by weight of the $200^{\circ}-500^{\circ} \mathrm{C}$ fraction. Carbazoles and their higher benz analogues also appear to account for a major part of the nitrogen in the $500^{\circ} \mathrm{C}$ plus residues from these crudes. Among the benzcarbazoles the 1,2-isomers predominate, the 3,4-benzcarbazoles are present in minor amounts, and the 2,3-benzcarbazoles are altogether absent. A similar distribution has been noted in catalytically cracked gas oils².

An examination of the possible origin of these important nitrogen compound types is worth while because it might provide indirect evidence on the origin of petroleum itself. The indole nucleus is found in Nature in the amino-acid tryptophan and many of the alkaloids ${ }^{3,4}$, while the hexahydrocarbazole nucleus exists intact in the alkaloids strychnine and aspidospermine. Similarly, as indicated in Fig. 1, certain natural alkaloids appear structurally related to the 1,2-benzcarbazoles, and, in a less direct fashion, to the 3,4-benzcarbazoles. Interestingly, no alkaloids known to the author possess the potential 2,3-benzcarbazole nucleus. It is striking in this connexion that the relative probability of producing a benzcarbazole from the naturally occurring alkaloids (that is, 1,2-benzcarba-most likely, 3,4-benzcarbazoles less likely, and 2,3benzcarbazoles unlikely) is precisely reflected in the actual occurrence of the various benzcarbazole isomers in petroleum. In the absence of alternative explanations for the relative occurrence of the benzcarbazole isomers in petroleum, this suggests that these petroleum nitrogen compounds were formed from ancient plant deposits. The preference for the angular 1,2- and 3,4-benzcarbazole structures in petroleum also parallels the general preference in Nature for angular as opposed to linear structures (for example, the steroids).

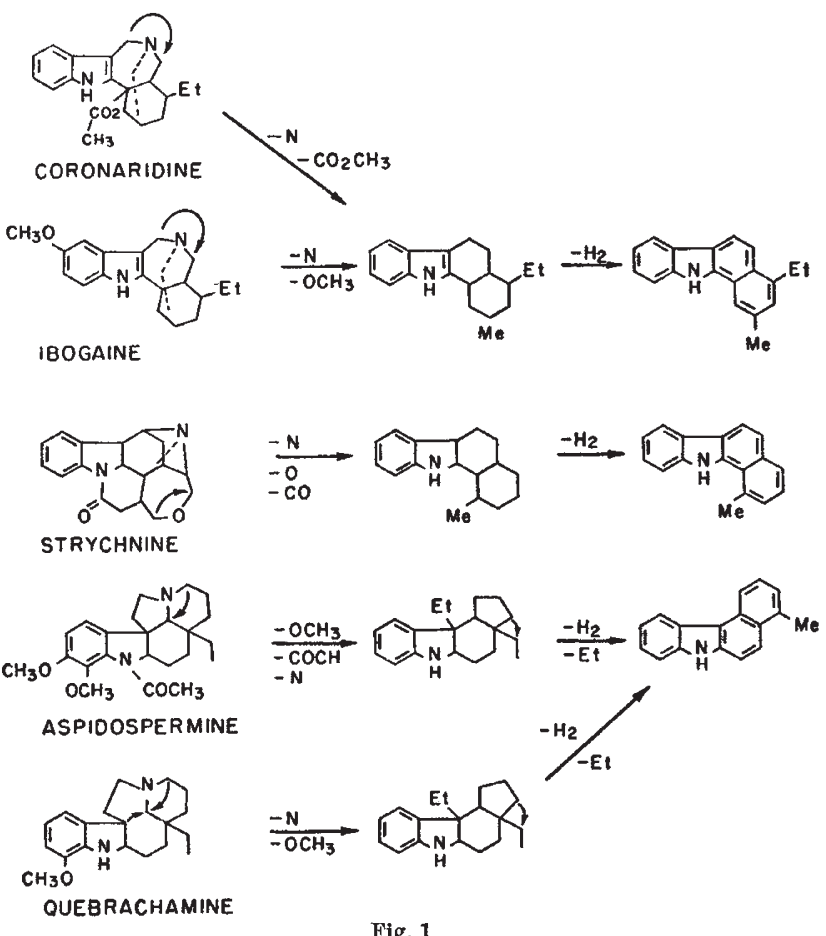

Fig. 1

The relative absence of the indoles in petroleum ${ }^{1}$ can be rationalized in terms of the much greater reactivity of this class of compounds toward acid and other environments (for example, ref. 2).

Union Research Center,

Union Oil Co. of California, Brea, California.

1 Snyder, L. R., and Buell, B. E., submitted for publication in Analyt. Chem.

'Snyder, I. R., and Buell, B. E., Anal. Chem., 36, 767 (1964).

s Sumpter, W. C., and Miller, F. M., in The Chemistry of Heterocyclic Compounds. Heterocyclic Compounds with Indole and Carbazole Systems, edit. by Weissberger, A. (Interscience, New York, 1954).

- Biemann, K., Mass Spectrometry. Organic Chemical Applications (McGrawHill, New York, 1962). 